\title{
Asymptomatic Visceral and Renal Artery Stenosis in a 49-Year-Old with Aortic Iliac Occlusive Disease
}

\author{
MODERATOR: KEITH D. CALLIGARO, M.D. \\ PANELISTS: $\quad$ ENRICO ASCHER, M.D. \\ WILLIAM D. JORDAN, M.D.
}

ABSTRACT We will present a 48-year-old man with disabling bilateral lower extremity claudication caused by diffuse aortoiliac atherosclerotic disease. The arteriogram documented three-vessel visceral artery occlusive disease and unilateral $60 \%$ stenosis of the left renal artery. The focus of the discussion will be the preoperative and intraoperative management of this patient, especially the role of prophylactic mesenteric revascularization at the time of aortic surgery.

Keywords Prophylactic mesenteric revascularization, aortioiliac occlusive disease

Moderator: This patient is a 48-year-old white man with a chief complaint of bilateral leg claudication. His symptoms began about 9 months ago. He developed left buttock aching that radiated down the entire left lower extremity, including the thigh and calf, after walking one block. In addition, he has developed right calf claudication after one block. He denies any right buttock or right thigh aching. The symptoms are clearly worse on the left than on the right and have been worsening over the last 9 months. He stated that for the last week or so he was starting to get some paresthesias in the left foot at night, although it was not clear if the discomfort was really rest pain or not. He also has been impotent for two years. He has smoked two packs

K.C., Chief, Section of Vascular Surgery, Pennsylvania Hospital, Philadelphia, PA. E.A., Professor of Surgery, SUNY Health Center, Brooklyn; Director Vascular Surgery Services, Maimonides Medical Center, Brooklyn, NY. W.D.J., Associate Professor of Surgery, University of Alabama Hospital at Birmingham, Birmingham, AL.

Copyright (C2000 by Thieme Medical Publishers, Inc., 333 Seventh Avenue, New York, NY 10001, USA.

Tel. +1 (212) 584-4662. 0894-8046,p;2000,13,1,85,102,ftx,en; pvs000094 
of cigarettes a day for 35 years since he was 13 years old. He has had hypertension for 2 years, but it is well controlled only on one medication. He denies diabetes or hypercholesterolemia. He denies myocardial infarction or angina, stroke or deep vein thrombosis. He denies any abdominal pain after eating and has not had any weight loss, although he is very thin. In summary, his only symptoms have been difficulty walking after one block.

On physical exam he is a thin, white man. There is a left carotid bruit. His heart had a regular rate and rhythm without murmurs; lungs were clear. There were no abdominal bruits or abdominal masses or tenderness. He had a $1+$ right femoral pulse and no palpable distal right leg pulses. There were no palpable pulses in the left femoral artery or distally. There were no ischemic lesions of the feet and no dependent rubor.

His noninvasive studies revealed a systolic blood pressure of $90 \mathrm{~mm} \mathrm{Hg}$ in his right high thigh and $75 \mathrm{~mm} \mathrm{Hg}$ in the left high thigh. Both ankle pressures were $75 \mathrm{~mm} \mathrm{Hg}$. An ankle/brachial index was not accurate because his brachial artery pressures were $75 \mathrm{~mm} \mathrm{Hg}$ bilaterally. The pulse following recordings in the high thighs were diminished, especially on the left. After exercise on a treadmill, his ankle pressures decreased to zero and the pulse volume recordings in the ankles became totally flat-line and remained that way after 15 minutes of resting. They improved after that period. The studies were consistent with severe aortoiliac occlusive disease and severe bilateral lower extremity claudication.

In summary, this is a young, apparently healthy, 48-year-old man with severe disabling bilateral leg claudication. He smokes and has mild, easily controlled hypertension. He had a $1+$ right femoral pulse but no other pulses in either lower extremity, and his noninvasive studies were consistent with severe claudication. Dr. Ascher, would you like to comment?

Dr. Ascher: I would like to make a couple of points and comments, just before getting into the options of treatment: (1) Here we have a 48 -year-old gentleman who is not in any acute distress despite all these horrible, noninvasive and invasive testing results. He is a claudicant for one block and he continues to smoke which bothers me a lot; (2) He also has a left carotid bruit that also bothers me. So, I would ask two questions: whether he has the potential to stop smoking and whether he will exercise, and whether he was placed on any pharmacological therapy for claudication. I didn't get that in the history.

Moderator: Because of persistent emphasis to stop smoking from his primary care physician, he quit smoking about 2 months ago. He hasn't noticed any change in his leg symptoms. At this point, medications were not tried. Would you recommend medications in this young patient?

$D r$. Ascher: At this point I would recommend exercise therapy and maybe a form of "Pletol." That is what I would start with initially. I would not have obtained an angiogram, just stopped at the PVRs actually, and I would have discussed with the patient that his long-term outlook is not great if he goes back to smoking, which is a very common finding in these patients. I would 
emphasize that. I would place him on an exercise therapy, somewhat controlled, if there is a center close by to his home I would send him there. There are a couple of centers that concentrate on improving a patient's claudication distance with controlled exercise therapy on a treadmill. They go three times a week. It appears there is some hope for this patient so I would start with the exercise therapy and, of course, I would follow him closely to see whether his disease is evolving.

Modevator: How long would you persist with the exercise therapy, Enrico, to see if there has been improvement, and with the "Pletal" -2 months?

Dr. Ascher: Approximately 2 months.

Dr. Jordan: A couple of things I would add. I would get a duplex image of his cerebrovascular system and try to image the subclavian; I would assume he has some subclavian disease if you have a functioning patient with a pressure of $75 \mathrm{~mm} \mathrm{Hg}$ in both arms. I would do that as a baseline.

I agree pretty much with the initial medical course; I would probably be a little harsher with him, try to scare him away from the cigarettes. He just stopped and as long as you can secure that, but add "Pletol" as suggested, and a walking program. I usually want to go at least 3 months before I can assess the efficacy of "Pletal." I would say 3 months with this and return with another walk on the treadmill.

Moderator: We obtained a duplex scan of the carotids and noninvasive studies of the upper extremities also. The duplex was consistent with $<50 \%$ stenosis of the left internal carotid and minimal stenosis of the right internal carotid. The duplex scan and upper extremity noninvasive studies suggested severe stenosis or occlusion of both subclavian arteries.

Dr. Ascher: It could be that the innominate also is stenotic? Or were you not able to pick up any evidence on the study of the right carotid?

Moderator: The duplex did not suggest inflow stenosis of the innominate or the proximal common carotid. Nonetheless, he was being treated with one medication for hypertension. He was given "Pletal" and told to exercise three times a week and walk as far as possible. Three months later his symptoms had not improved at all. Follow-up noninvasive studies were also not improved 4 months later. By this time, he was somewhat upset that 3 months after his initial evaluation he was not walking any better and he could not work. At this time, he clearly wished something more definitive be done. A contrast arteriogram was planned.

Dr. Ascher: One other question, would you consider performing an MRI study prior to the invasive contrast arteriogram for assessment of the aortic segment that you suspected by the noninvasive testing to be diseased?

Dr. Jordan: Generally, no. Because to me the therapeutic decision is made based on the physiologic assessment that I get from the treadmill and noninvasive tests. I am going to assume there was no renal or visceral stenosis because he was essentially asymptomatic up to this point. So, I would have 
gone with the treadmill test and stopped there, and encouraged him in a medical program. I think the patients with more proximal disease, segment I, aortoiliac disease, are less likely to benefit from the medical program, but I generally prefer to start that way, so I would go with the treadmill, walk him, and see him in 3 months. He may come back anxious to have something done and then I would get more invasive with the evaluation.

Moderator: So, Dr. Jordan, you would get an arteriogram rather than a magnetic resonance angiogram (MRA)?

Dr. Jordan: I would obtain an arteriogram once I've made the decision to revascularize. If an endovascular option is available at that time, then I would consider it then. Of course, the decision to undertake balloon angioplasty and stenting versus open surgical reconstruction depends on the patient's overall medical condition, their expectations for therapy and the anticipated durability of therapy.

Modevator: Enrico, you bring up the MRA because that is what you routinely do, or because you are concerned about his baseline renal function. By the way, his creatinine was $1.2 \mathrm{ng} / \mathrm{mL}$.

$D r$. Ascher: No, I just feel that more and more we are moving away from invasive studies on the diagnostic side, and the MRAs are now getting to the point where they are becoming very reliable. What we do for the aortic segment in our practice is to obtain a duplex arterial mapping of the aortic segment, and add an MRA to complement the studies so that both are noninvasive. We get most of the information necessary and then we discuss with the patient the options that we have. We would do the arteriogram only during intervention, for example, angioplasty with or without a stent. Then we injected at that time only. At this point we have gone away from preoperative arteriograms.

Dr. Jordan: We have used MRA, but I have been more impressed with the CT scan done with intravenous bolus injection. These images are equivalent or superior to the MRA that we have had, and the patients tend to appreciate the CT scan more than the MR. We still have some of those claustrophobics who don't like the "gun barrel" of the MRI and are willing to accept a more open CT scan.

Moderator: Enrico, do you think the MRA would have helped you that much or would it have just added to the cost because you would have ultimately needed the arteriogram to perform a balloon angioplasty?

Dr. Ascher: That is a very good question. For most parts, if you review the MRA with the radiologist who has performed it, you can get much more information than just getting the picture. First of all, what you see on the screen - the patient-is clearer to you than if you just get the printout; there are different ways to see it, on transverse cut and on longitudinal (sagittal) cuts. So you will be able then to assess the segment of stenosis and actually measure that area, much more precisely than the radiologist eye balling it and telling you that there is a moderate or mild stenosis, with a severe stenosis. 
You can actually measure that on transverse cuts just by giving "galilinium," which studies enhance tremendously. You will be unable to differentiate a nine, $80-85 \%$ stenosis, but you will see a severe stenosis from one that is $60 \%$ or less. If the patient is symptomatic and you have noninvasive testings that are contributing to your understanding of this whole problem on this patient, and then you add collesion on the MRA that appears to be severely stenotic, you should feel comfortable. Now we feel very comfortable with a duplex scan also, particularly for noncalcified vessels. We are able to see and assess hemodynamically the lesion, specific lesions, the length of the lesion in ways we were not able to do before, so it has helped us a lot. Diverting a little bit from that I would like to ask you whether the arteriogram, biplanar, we have seen cases where also in the labs you did an angioplasty on one lesion and you still found that the PVRs were not up that great despite an excellent completion arteriogram. Have you see this?

Moderator: Yes.

Dr. Jordan: I agree. We have an underlying understanding that the angiogram underestimates the disease. I think an MRA can sometimes overestimate the disease, particularly in carotid disease. But an angiogram is an underestimation of disease and sometimes the balloon therapy can improve the angiogram; it does not always improve the symptoms.

Moderator: At this point, a contrast arteriogram was obtained that showed that there was an $80 \%$ stenosis of the celiac artery and $90 \%$ stenosis of the superior mesenteric artery on the lateral aortogram. There was also a $60 \%$ stenosis of the left renal artery and a widely patent right renal artery. The infrarenal aorta appeared normal immediately distal to the kidneys and then gradually became more diseased with atherosclerotic changes in the distal aorta. The inferior mesenteric artery had a $60 \%$ stenosis at its origin. There was clearly a very large meandering artery of Riolan that was perfusing the superior mesenteric artery. There was a $3-\mathrm{cm}$ long, $70 \%$ stenosis in the right common iliac artery and a $3-\mathrm{cm}$ long, $99 \%$ stenosis of the left common iliac artery. Both external iliacs were also diffusely and severely stenotic. The common femoral arteries were patent as were the superficial femoral arteries. Enrico, would you consider performing multiple balloon angioplasties in a 48-year-old man with diffuse disease like that?

Dr. Ascher: Yes. This man is a 48-year-old, but his arteries are of a 98 -yearold patient. So, I would not be misled by the fact that he is 48 years old. He most probably has severe coronary artery disease. He is just not symptomatic because he is not walking enough.

Moderator: I am mostly concerned about the fact that he may be more hypertensive than we think and there is no way we can really find out for sure, having bilateral subclavian stenosis, so this patient might be much sicker than we think he is. So, the age of 48 would not give me any assurance that he is a healthy young man. Therefore, I feel that a minimally invasive procedure for him will be the way to go, however, I have some reservations about 
the distribution of the disease in the iliac artery, particularly at the external iliac on the right where I see a stenosis that is very close to the groin. Those lesions have not been optimally dilated and they tend to have a high recurrence rate and become an iliac or proximal iliac, so the ones behind the groins are the ones that I have had most trouble with. Because most of these lesions cannot be stented I end up sometimes with results that are not optimal. But I would still attempt, first, a left iliac-common iliac and right common iliac most probably using $4 \mathrm{~cm}$ kissing balloons. I would go up the right groin also. I will obtain a completion angiogram, biplane, and if I am happy with that I will better assess then the lesion, the long one at the junction. I am not sure it is the junction or just all external iliac. I have those slides here that I am not so sure of. But, the external iliac on the left side appears to have a long stenosis. If that is above the groin I would also dilate it, stent it, and measure pressure. I will basically dilate all the lesions and if I have any question or the lesion on the right is not optimal because it is behind the groin, I will be prepared to do a fem-fem bypass. I would try to stay away from this patient's belly. We can discuss that a little bit more, right?

Modevator: Will, what about your impression of performing balloon angioplasty of his common and external iliacs?

Dr. Jordan: I want to be more aggressive. In the face of bilateral common iliac stenosis, multi-segment disease including the common femoral arteries, I would discuss with him the endovascular approach, but would lean toward an operative approach. Although he is chronologically 48 , I think physiologically he is probably 68 to 70 . I expect he needs 15 to 20 years of results to survive and while I would discuss with him balloon angioplasty, he has long enough lesions that I would give him more definitive aortofemoral reconstruction at this time.

Dr. Ascher: Will, do you think this man has an average survival of 20 years?

Dr. Jordan: No I don't. The 5-year survival rate for claudicants runs about 60 to $70 \%$ and so I want to give him a result that will let him last his lifetime. So, I want to give him a 10 -year result. That might be a better way to say it. I think I have a better possibility of giving him a 10-year result now with an aortofemoral. Of course, I would screen his heart ahead of time, too, but I want to give him a definitive reconstruction at this point and save other options for later. I think aortofemoral has great long-term patency and it is probably the time to do it for him.

Moderator: Enrico, are you implying that anyone under 60 years old with diffuse aortoiliac disease should have balloon angioplasties performed before an aortobifemoral bypass?

Dr. Ascher: I may have missed (assuming the balloon angioplasties could be done)-reviewing the literature on what is the average survival of patients who present with such aggressive virulent atherosclerosis who are under the age of 60 , compared with patients over the age of 60 , but if I have to take a guess I don't think it is that much better. In addition, I believe the 
aortofemoral bypass for aorto-occlusive disease has at best a 60 to $70 \%$ patency because of progression of the disease at the groin, and in the SFA and profunda.

Modevator: A $60 \%$ five year patency?

Dr. Ascher: I would say 60 to $70 \%$, for aortofemoral occlusive disease. On patients with extensive atherosclerotic disease these patients are not the common variety of single lesion, you know hypertensive.

Moderator: The SSAs are open?

Dr. Ascher: Yes, they are. He has a proximal stenosis on the right SSA, but otherwise they are open.

I would have to review the current data on aortofemoral, but I wouldn't say it was 80 to $90 \%$. On the other hand, a balloon angioplasty in the iliac system is reported to be up to $75 \%$ patency at 5 years and better with stents, so I am not so sure that it is that much better. I mean the operation is that much better than balloon angioplasty and stenting the iliacs. I also would be cautious about the extent of iliac angioplasty.

Modevator: I would agree with Dr. Jordan. I have become less enthusiastic about endovascular treatment for diffuse aortoiliac disease, especially when the external iliac arteries are involved. Our vascular surgeons here at Pennsylvania Hospital have been performing iliac balloon angioplasties for several years and we have been extremely disappointed with the long-term results of external iliac balloon angioplasty. And for that reason, we discussed that as an option, however, I clearly told him that I would favor a more aggressive approach, which is what he was more in favor of also.

Dr. Ascher: What if you had done the angioplasty with a stent and waited 2 to 3 years? What have you basically lost? You have to agree that there is a possibility that it may work, long-term.

Dr. Jordan: I would agree with that, Enrico. I think it is very acceptable to go with a balloon approach first, but the ones that I think have the most success are the focal segmental common iliac lesions, not the ones that are long and diffuse. You now have an angry patient because he has endured 3 to 4 months with minimal improvement and he wants definitive improvement. That is why I would be more aggressive, rather than take an intermediate approach.

Dr. Ascher: The reason is, not that I do angioplasties every day, and not arterial constructions, but I am mostly concerned about the distribution of his mesenteric circulation. I am a little bit concerned about clamping the aorta without addressing his mesentery vessels, the chance of damaging the mesenteric circulation is real. I think the chance of embolizing from such an atherosclerotic aorta is also real.

Dr. Jordan: Does your MRA routinely evaluate the mesenterics and the renals? Are the results accurate?

Dr. Ascher: No, we don't. The MRA is very accurate. And for the viscera an MRA is excellent, really excellent. 
Dr. Jordan: So, if you were basing your decisions on MRA and noninvasive studies, you would know ahead of time that he had pretty extensive visceral and renal disease, or moderate renal disease.

Dr. Ascher: I will miss the SMA lesion and celiac lesion. I will see the renal. I am not so sure that I will be able to see the superior mesenteric artery very well. I have not had experience with visualizing the superior mesenteric artery; again, it is in the last 6 to 8 months that we have moved into the MRA arena.

Moderator: To put the iliac balloon angioplasty issue to rest, or possibly add more controversy to the issue, I think there is maybe a downside to attempting a balloon angioplasty in this gentleman. I don't think the patency rates are going to be $75 \%$ at 5 years with such diffuse disease. For a short, isolated common iliac stenosis, the best patency rates are this good. In Johnson's multicenter Canadian study (JVS 1992), he reported a 60\%, 5-year patency rate. In addition, there is a small but definite potential that balloon angioplasty of such diffuse disease can result in acute arterial thrombosis and/or perforation. To then convert this gentleman to an emergent abdominal procedure, which would be necessary probably in less than $5 \%$ of cases, is of concern to me without having evaluated his cardiac function. But you bring up a very good point in that his long-term survival may not be that great either.

Dr. Ascher: I would say that if you were concerned about an angioplasty because of his heart you certainly would be much more concerned about an operation. But all these points are clear and it is a very interesting case to discuss because there is real lack of published data to tell us exactly what is the best way to go and I think every patient has to be discussed in the way we are discussing.

Moderator: So, let's say that we either try the balloons and the stenosis recurred, or we just felt we needed to be more aggressive from the start. All three of us would be of the opinion that he needs some type of cardiac testing. Will, at the University of Alabama, what test would you recommend?

Dr. Jordan: I routinely use echo first, plain echo, no "dobutamine," but just a plain echo. I probably would obtain a "Persantin thalium" additionally, although if I have a pristine echo and normal EKG and no symptoms, sometimes I proceed with surgery. It is partly because he has great vessel disease also, that is the reason to go to a thalium. So, if I get a combination of thalium and normal echo, then I will proceed from there.

Dr. Ascher: We use thalium for aortic operations. I am not so sure that it is really that worthwhile because I am not certain whether a coronary angioplasty on this patient or a coronary bypass has been proven so effective as we have been led to believe. I don't think we have a single good study showing that fixing this patient's heart actually has initial impact on the results of our operations. But, at this point we are still doing it for medicolegal reasons; I am sure you all know about the recent paper in the New England Journal of 
Medicine stating that patients on betablockers did very well after vascular surgery, despite severe coronary artery disease, just by treating them with betablocks. So, I think at this point we are trying to be clinicians and ask our cardiologists to think about using betablockers, more so than Di- pyridamol, thalium and invasive treatment for coronary artery disease.

Modevator: All three of us are in agreement that this gentleman should probably get some type of a stress test. We performed a Persantine stress test and as expected it was markedly abnormal. Our cardiologists recommended that he have a cardiac catheterization. Would you agree with that course of action? Or would you instead tell him, "You came to us with claudication. Now you are facing a cardiac cath and possible CABG, even before we subject you to a major operation to help you walk better," and therefore recommend to him he not undergo a cardiac catheterization and just live with his claudication and retire from his job?

Dr. Jordan: Go get the cath, get it fixed, you're there.

Dr. Ascher: Now we cannot say not to get a cath.

Modevator: As expected, the cardiac catheterization showed severe triple vessel disease. He underwent a successful three-vessel coronary artery bypass.

$D r$. Ascher: May I ask a question? Did you mention or have the opportunity to mention to the cardiac surgeon not to use the IMA as a source of inflow vessel?

Modevator: Yes, I did mention that because we were concerned about collateral dysfunction to the legs and maybe into the gut somehow. He came through the CABG successfully. One month later he had fully recovered and felt strong enough to undergo aortic surgery. Any other comments?

Dr. Ascher: I have one. Did you consider doing, at the same time, an ascending aortobifemoral bypass while they had the chest open? Because, that would entail only an addition of two cutdowns in the groin. The aorta was right there and that would avoid the entire complex abdominal operation.

Modevator: I did not consider that.

$D r$. Ascher: We had experience with two patients. It was very, very simple because the only thing we had to do was perform an anastomosis in the groin and we tunneled the grafts subcutaneously, under the sternum and subcutaneous tissue, and this worked very well, a bifurcated graft actually. I'm sorry, it was a straight graft and we did a fem-fem.

Moderator: That is an option to keep in mind. However, you are then limited in performing any visceral or renal revascularization, which we'll get to in a minute.

Dr. Jordan: Did they image his arch at the time of the cath?

Moderator: Yes. The study showed bilateral subclavian artery stenoses and/or occlusive disease, but his innominate was all right.

Dr. Ascher: Proximal to the IMA, takeoff?

Moderator: On the left side it was actually the axillary artery that was diseased. His IMA was all right. 
Dr. Ascher: It would be interesting, because if it was proximal and you can fix that, it may improve circulation to the leg.

Moderator: Assuming that we are at the point where we agree he should have an aortobifemoral bypass, I would want you both to consider the following scenario. If the distal external iliac and common, superficial and deep femoral arteries are widely patent on the preoperative arteriogram, and the distal external iliac arteries feel soft and minimally calcified when the abdomen is open, would you consider performing a bypass from the aorta to the distal external iliac arteries in the abdomen to avoid the morbidity associated with groin incisions? Do you feel it is mandatory to always go down to the groin and anastomose to the common femoral arteries?

Dr. Jordan: I am going to say, mandatory about $95 \%$ of the time. It is partly because I haven't found one. Even one with a soft artery, that external iliac is thick and the planes of the artery separate differently. I have reserved that for aneurysm disease and pretty much avoid it for occlusive disease.

Dr. Ascher: I think most of the external iliac arteries will be very diseased in these patients and the ones that have less disease, then they would be candidates for angioplasty.

Moderator: In our experience, there have been several patients whom we have been able to anastomose to the distal external iliac arteries through an abdominal incision and thereby avoid groin incisions. We believe that if the distal external iliac arteries are soft and the arteriogram shows that distally the arteries are widely patent, we favor doing a bypass to the external iliacs as distally as we can in the abdomen. We realize that progressive disease may occur in that 1- or 2-inch long segment distally. On the other hand, most graft and wound infections are associated with groin incisions.

Dr. Jordan: I think the groin complications occur in the fat patients and those are harder to get to the external iliacs. A thin patient like you have here, I think the groin complications are miniscule.

Dr. Ascher: I would tend to agree with Keith because we preferentially use the external iliac artery for inflow for re-do groins. It is interesting how often the artery is softer than we think, however, because we are doing so few aortofems or aorto-iliacs, we just don't see these patients anymore. Most patients now, I don't know in your practices, but in my practice we do fewer than eight, anywhere from 5 to 8 aortofemorals out of 1500 cases, so we see fewer and fewer because of the angioplasty and stenting. You have a very good point about not crossing the groin. The chance of false aneurysms in the next 10 years would be still around $5 \%$ and the chance of infection is shown over and over to be higher in the groin than when you keep the graft in the abdomen. That is why we all attempt, during an aortic aneurysm repair, to keep in the graft in the belly.

Moderator: The next question pertains to the proximal aortic anastomosis. Do you think in this patient you would do an end-to-end aortic anastomosis or would you consider an end-to-side? 
Dr. Ascher: My plan for this patient will be to do not only aortobifemoral bypass but also extend into the SMA. I do most of my anastomosis end-toside. I feel that an end-to-side has an advantage when the graft closes that the native circulation is still better than when we do end-to-end. I also feel that although he is totally asymptomatic and I probably wouldn't have done anything if I had to stay away from the aorta, I feel now that if I am there I should fix the SMA just because I don't know what the outcome will be if I don't, if I just leave him alone.

Moderator: So you would do an end-to-side proximal aortic anastomosis and a limb to the SMA. The IMA had a $60 \%$ stenosis at its origin. Would you just leave that alone? Will, what about end-to-end or end-to-side proximal aortic anastomosis for occlusive disease?

Dr. Jordan: I would do an end-to-end, almost universally, and if the IMA looks important to me, and in the face of this three-vessel visceral disease I would do an end-to-end where I implant the IMA and at the same time bring a little jump graft off and bypass the SMA. I am willing to reimplant two of the viscerals while I am there. I prefer end-to-end, I think the flow pattern is better. I like the way, after cutting out a segment of the aorta, you can drop it down to the same anatomic plane so it is easier to cover and has a theoretic risk of reducing intestinal fistula.

Moderator: It is easier to cover the graft with some type of soft tissue over an end-to-end configuration than an end-to-side graft. I would propose that current recommendations many vascular surgeons offer to trainees is that an end-to-side anatomosis is preferable when maintaining patency of the terminal aorta and common iliacs to provide blood supply to the internal iliacs arteries. If the external iliacs are totally occluded and there is only moderate or mild disease in the aorta and the common iliacs, then an end-to-side proximal aortic anastomosis should be performed to perfuse the internal iliacs. When the terminal aorta and common iliacs are severely diseased and/or occluded, and the external iliacs are patent, then an end-to-end proximal aortic anastomosis is preferred because there will be perfusion back up the aortobifemoral graft into the external iliacs into the internals to maintain pelvic circulation. Do you two generally agree with that?

Dr. Jordan: I generally agree. There are some additional options when you want to preserve a hypogastric flow and I will even take my initial limb of aorta-femoral and drop it to the common iliac, the distal common iliac, to provide hypoastric flow and then piggy-back from the limb of the graft to go down to the femoral.

$D r$. Ascher: I think the end-to-side anastomosis would not provoke any decreased flow distally. It doesn't make any sense to me to transect the aorta to improve pelvic circulation. I don't see how that would occur.

Modevator: Would you expect this gentleman's impotence to be better after an aortobifemoral bypass? 
Dr. Ascher: If this was a very ischemic problem, we have not proven this. Had you performed any noninvasive testings?

Dr. Jordan: I would not have routinely done that. Revascularization will be improving his hypogastric flow and retrograde flow back up the external iliacs can provide that. But I also will be doing at least partial dissection around the bifurcation, so he has the potential of even a retrograde ejaculation, because of the dennervation that may occur. I would essentially tell him that we are not undertaking this operation to improve impotence. We have Viagra, or a urologist can help with something else.

$D r$. Ascher: It is possible this patient may be impotent, ischemic in origin. I think that a full workup preop will not be a bad idea.

Moderator: I don't disagree doing the penile testing. The reason we didn't do it was I didn't think his impotence would get better because both internal iliacs arteries were essentially occluded on the arteriogram, although the left one may be slightly patent. My point was that because the internals were essentially occluded, I wouldn't expect his pelvic perfusion to improve. Therefore, I told him that I thought the chances of his impotence improving were nil, but if it got better, I would take credit for it.

Dr. Jordan: Are you willing to drop a graft down to his hypogastric?

Modevator: On the arteriogram, I didn't see much of an internal iliac on either side. No, I wasn't willing to do that.

The primary reason for presenting this case was prophylactic visceral and renal revascularization in a young person with aortoiliac occlusive disease. Would either of you consider doing a prophylactic bypass to the left renal artery? He is on one antihypertensive medication and it appeared to be about a $60 \%$ left renal artery stenosis.

Dr. Jordan: I want two other tests. I want to know the central aortic pressure at the time of catheterization and then I would likely do a Captopril renal scan, too. I am going to assume the Captopril renal scan is marginally positive and then I'd probably do a renal bypass at the same time.

Moderator: Assuming his blood pressure, based on his central pressures, was higher than you expected and it wasn't well controlled, and the Captopril scan was positive, you would do an end-to-end aortic anastomosis and bring both limbs down into the groins, reimplant the IMA onto either the distal stem of the graft or the left proximal limb of the graft, and then do a jump graft from the stem of the graft to the left renal artery. And you would use a prosthetic graft for the renal, probably, a $6 \mathrm{~mm}$. Dacron. Now, also off the stem of the graft you would do a C-loop back to the SMA? Enrico, would you do a left renal prophylactic bypass, assuming his essential pressure was higher than you expected and assuming the Captopril scan did show a somewhat abnormal on the left side?

Dr. Ascher: First of all, I would be surprised if the results would be abnormal. Second, there is not really evidence for this patient who is practically asymptomatic with the contralateral artery being patent and undiseased, to 
believe that we could really help him doing a bypass to his renal artery on the left. But I really feel there is not much evidence to perform an additional operation. I would be concerned about extending operative time and adding so many bypasses to a gentleman who just had open heart surgery and is not too healthy. Primarily, I feel there is no evidence to support doing a prophylactic renal bypass. I know it is a very controversial subject and there are groups who are much more aggressive and there are ones who take a much more observant approach and I belong to the latter group, at this point with a $60 \%$ unilateral stenosis. You could make a much stronger point if this patient had bilateral severe renal artery stenosis.

Even if he was not heavily symptomatic I would certainly perform a renal bypass to the one most stenotic. We are already doing a bypass of SMA and both femoral arteries, I would probably stop there.

Dr. Jordan: One addition, I think the latest natural history paper was about $5 \%$ per year in terms of progression of stenosis. My feelings on revascularization on the renal artery are probably not as strong as on the visceral on this patient. The left renal is relatively easily accessible, based on what we are doing on the aortic dissection; it is a whole lot easier to do it during this operation than it is to come back later. I do recognize the potential of an extraanatomic, splenorenal bypass-or even an endovascular approach can also be undertaken. Also you "open the door" for angioplasty and stenting if you do an aortofemoral graft for him. My thought is if the pace of the operation is going well, the renal bypass would be the last on my list, but I would still proceed with it. It is just one additional anastomosis to do while heparin is already circulating.

Modevator: Now to the visceral part. You both would do a jump graft to the superior mesenteric artery. Enrico, you would not do anything to the IMA, but Will, you would reimplant it into the side of your graft?

Dr. Ascher: I would not. I would just go above it, just below the renals. One way to avoid the bulky graft of this is to perform a long anastomosis. I have never had any problem closing the peritoneum over the graft. As long as we don't make a long knot that ends up sticking through the peritoneum to the bowel, we try to bury that posteriorly, almost toward the spine, and you pretty much cover this problem.

Modevator: I think very few vascular surgeons would recommend a pure prophylactic mesenteric revascularization if you were not going to be in the abdomen for another reason. Are we sure or reasonably sure that it is worth it to do a prophylactic mesenteric revascularization when you are doing an aortic bypass? Enrico mentioned the natural course of renal artery stenosis. Is mesenteric artery stenosis that much more progressive?

Dr. Jordan: There was a paper at the Peripheral a year or two ago in Annals of Vascular Surgery that showed visceral reconstruction was durable at relieving symptoms and also durable in terms of its long-term patency. 
Moderator: Absolutely right, but the question I want to address is an asymptomatic patient who has visceral artery stenosis, and you were there for either an aortic aneurysm or occlusive disease. I am going to go on the assumption that visceral reconstruction is durable with good patency numbers. The next point is, this guy has three-vessel disease, not like you are dealing with a median arcuate ligament on someone. He is thin and you can't assume any malnutrition or weight loss, but he is a sort of a setup for the patient who is going to suddenly occlude one of these and not have the collateral flow to survive. Again, you are there at the time doing the aortic reconstruction, one additional graft (you can argue two in my case if I am going to drop the IMA in there it is going to increase the time) I don't think it is going to increase the morbidity considering the potential this guy has in facing mesenteric problems in the future.

Dr. Ascher: The problems in patients with peripheral arterial disease are not well known. I remember there was a paper from VA where they attempted to find out what the problem was. They came out, I don't remember the number exactly, I think about $30 \%$ of the patients had actually a $50 \%$ or greater ciliac or SMA stenosis and a little less than $5 \%$ had significant stenosis in both mesenteric arteries. So, it exists. Perhaps we are not looking and studying it that much, but it exists.

So, I think for the stenosis there, the fear that it will close and cause the patient to be symptomatic has not really been proven.

Moderator: I do not believe there is much in the literature to help us out regarding prophylactic mesenteric revascularization. There are several series showing that you can do it and obtain good patency rates, but that is not the issue. The important point is what happens if you don't prophylactically revascularize diffuse mesenteric stenoses when you're operating for aortic aneurysms or aortic occlusive disease. A series from Emory published in JVS in October, 1988 (Stanton PE, Hollier PE, Seidel TW, et al. J Vasc Surg 1986;4:338-344), reported mesenteric revascularization in 41 patients, ten for prophylactic reasons at the time of aortic surgery. All ten patients did well and they remained asymptomatic. That's great. But that argument is similar to arguing that you can operate on asymptomatic $30 \%$ carotid artery stenosis and all those patients do well. That's not this issue.

Dr. Jordan: That's not quite the same, remember I was Emory trained.

Moderator: The other thing to keep in mind is the group from Emory are excellent surgeons who get good results with very complicated surgery.

Another paper from Connelly in California in the Annals of Surgery 1979 (Connelly JE, Kwaan JHM. Ann Surg 1979;190:514-521) also showed that prophylactic visceral revascularization at the time of aortic surgery is safe, but to me that is not really the issue. Another paper I want to mention was published in Journal of Vascular Surgery in May, 1998 from the University of Kansas (Thomas JH, Blake K, Pierce GH, et al. J Vasc Surg 1998;27: 840-844). They obtained arteriograms on all patients being considered for 
aortic surgery and found 72 patients with more than $50 \%$ stenosis of a visceral vessel. Twelve patients were omitted who only had stenosis of only one visceral vessel. Of the remaining 60 patients who did not have prophyactic mesenteric revascularization, four $(6 \%)$ went on to develop mesenteric ischemia. One could make the argument that the operative mortality for elective visceral revascularization is $5 \%$ anyway and therefore you are really not helping these patients. If you specifically focus on the patients with severe stenosis or occlusion of all three mesenteric arteries, as in the patient we are discussing, 15 patients in the series from the University of Kansas met this criteria. Four of the 15 patients, or $27 \%$, went on to develop symptoms of chronic mesenteric insufficiency (3) or died (1) of acute mesenteric insufficiency. The authors' point is that if you have three-vessel disease, there is about a $27 \%$ chance that during the next 2 years patients will develop mesenteric insufficiency. Therefore, the authors propose that you should perform prophylactic mesenteric revascularization at the time of aortic surgery if there is three-vessel disease.

On the other hand, 27 of the 72 patients, or $40 \%$, died an average of 2 years later. Only one of them died due to mesenteric insufficiency. Because the long-term survival is so poor in these patients, should you worry about prophylactic mesenteric revascularization? The other side of the coin is that if the long-term survival is so poor, you probably shouldn't be doing an aortobifemoral bypass on them anyway. At any rate, I am not convinced that prophylactic mesenteric revascularization at the time of aortic surgery is worthwhile.

Dr. Ascher: I basically agree with what you said. My only concern is damaging the mesenteric circulation during the aortic procedure, and since I am there I might as well do it, but I certainly would not do it prophylactically, if I had another way to fix this patient's ischemic legs.

Dr. Jordan: I think you hit all the right points, the key being to be careful throwing this guy into pure prophylactic mesenteric reconstruction for the two reasons you stated: you're doing the aorta and this is a severe three-vessel disease.

Moderator: We performed end-to-end proximal aortic anastomosis. We placed the distal anastomosis into the common femoral and deep femoral arteries and also needed to do endarterectomies. As is often the case, the arteries didn't look too diseased on the arteriogram, but they were extremely calcified and very difficult to sew. We decided that because he had a large meandering artery of Riolan, revascularizing the IMA would be enough. The IMA anastomosis was performed as a Correl patch and reimplanted onto the side of the aortic graft. Doing all of this required 5 hours, mainly because his aorta and common femoral arteries were very calcified and diseased.

At that point, Will, since you were leaning toward still going ahead and doing the SMA, if it had taken 5 hours to do all of that, would you now add on the SMA bypass? 
Dr. Jordan: I would have constructed the graft beforehand and realized that I haven't overtly completely committed.

Moderator: At that point, you have done the aorto-bifem, the IMA.

Dr. Jordan: I have the SMA graft sitting there in front of me, because I sewed it before I ever dropped the graft in the belly. However, if it were 5 hours into the operation I probably would want to stop, that is getting to be enough. If I had already dissected the SMA or if it was straightforward I would like to put it on and I would put the renal to the end of that, so I would forego that renal, I think realizing that if this guy has more visceral problems later he would require a supraciliac aortic graft.

Dr. Ascher: If you had gone to the femorals primarily and not thought about the external, by angiogram you could see the external iliacs were very diseased, especially on one side.

Modevator: We did start in the groins, especially on the one side that looked very diseased all the way down to the groin.

$D r$. Ascher: Routinely I like to start at the groin, get a good idea of what the vessels look like and then open the belly. That would minimize the exposure.

Moderator: I agree that especially when there is diffuse disease going to the distal external iliacs or beyond, we start in the groins to minimize the time the belly is open.

Dr. Jordan: How did he do?

Moderator: He did well. He is still not smoking. He is walking as far as he wants. His right groin incision broke down, but the graft was never exposed. It took about 3 weeks for the wound to granulate. This case shows why I like to stay out of the groins if possible.

Dr. Ascher: Since you are the expert in groin infection, let me ask you, how would you manage an exposed graft? Say you went in and the graft was exposed. You certainly would debride widely; how would you manage the graft now?

Moderator: It wasn't pseudomonas. As long as the anastomosis was intact and not septic, I would try to preserve it with wound debridement, intravenous antibiotics, and muscle flap placement.

Dr. Ascher: You do a muscle flap.

Moderator: We prefer a gracilis or rectus abdominis flap if it is a small wound. If it is a big groin wound, then a rectus abdominis muscle flap.

$D r$. Ascher: It is just so much easier for us to use the sartorius, and because of that we have used or put our clinical experience together and we have been very happy with this aggressive approach in muscle transpositions in the absence of any infection- just cover the graft and close it routinely. We have done close to 30 cases.

Moderator: This is prophylactic coverage you are talking about?

Dr. Ascher: No, that involves a series of things. Just the fact that we are taking the sartorius more often than we used to in the past. In a couple of 
cases, sure, we had to go back and the distal portion of the sartorius was necrotic, not infected. Then we moved to the rectus abdominis which is a much bigger operation, and when it comes to that lateral flap then we usually get Plastic Surgery involved but the sartorius we just do it ourselves.

I think it is a well managed case, very complex, and I would agree with the steps you have taken. We have some difference in opinions because it is mostly the lack of literature, particularly in the mesenteric situation.

Dr. Jordan: The entire case highlights the aggressiveness of atherosclerosis. It should be classified as a neoplastic disease.

Modevator: Thank you both very much for your time and expertise. 
